\title{
Role of the Mammalian Target of Rapamycin (mTOR) Signalling Pathway in Podocytes in Glomerular Disease
}

\author{
Lena Succar, David CH Harris and Gopala K Rangan \\ Centre for Transplant and Renal Research, \\ Westmead Millennium Institute, The University of Sydney \\ Australia
}

\section{Introduction}

The key intrinsic cell of the glomerulus is the podocyte, which is described as the "organiser and caretaker" of glomerular structure and function (Leeuwis et al., 2010). Podocytes (also known as visceral epithelial cells) are terminally differentiated pericyte-like epithelial cells that form the outer exocapillary layer of the glomerular filtration barrier. They have a critical function in maintaining normal glomerular capillary permeability and functional integrity (Marshall and Shankland, 2007). The aim of this chapter is to summarize the current research on the contribution of podocytes to the disease process in glomerulonephritis in connection to the mTOR signaling pathway.

During glomerular development, the podocyte has a central role in integrating signals from the other glomerular resident cells to form the proper basic structure of the glomerular filtration unit (Eremina and Quaggin, 2004). Podocytes line the outer aspect of the glomerular basement membrane (GBM) and physiologically have a unique and complex cytostructure consisting of three morphologically and functionally distinct segments: a cell body, foot processes and the slit diaphragm (Mundel and Kriz, 1995). Major processes arise from the cell body and split into foot processes. The foot processes contain an actin-based cytoskeleton that is linked to the glomerular basement membrane at focal contacts (Faul et al., 2007). Podocyte foot processes form a highly branched interdigitating network which enwrap the glomerular capillaries, and are connected by the slit diaphragm. Three distinct membrane compartments form the foot processes: the basal side, the apical side, and the slit filtration diaphragm. The basal side connects the podocyte to the GBM with several types of integrins and dystroglycans (Pavenstadt et al., 2003). It also plays a role in endocytosis of albumin and IgG trapped in the GBM, offering possibilities for drug delivery (Eyre J, 2007) (Akilesh S, 2008). The apical side contains negatively charged proteins such as podocalyxin, a sialoglycoprotein, and podoplanin which form a glycocalyx layer and thereby contribute to charge-selectivity of the glomerular filtrate (Koop et al., 2008) (Orlando RA, 2001) (Kerjaschki D, 1984).

The slit diaphragm is the most complex layer of the glomerular filtration barrier, with a constant width of $40 \mathrm{~nm}$ (Tryggvason et al., 2006, Benzing, 2004). The main function of the slit diaphragm is to form a size-selective barrier for proteins and since many of the slit diaphragm proteins are phosphorylated, this contributes to the charge-selective element (Tryggvason K, 
2001). Podocyte-specific multiprotein complexes, similar to adherens junctions, extend between the filtration slits, closely interact with the actin cytoskeleton, influencing signaling pathways and motility of the podocyte (Lee et al., 2009) (Burt et al., 2007) and thereby establishing the final barrier to urinary protein loss (Somlo and Mundel, 2000).

The proteins which make up the slit diaphragm system are nephrin, Neph1, Neph2, podocin, fatty acid transporter tumor suppressor homolog-1 (FAT1) and FAT2, and the calcium channel transient receptor potential cation 6 (TRPC6) and cadherins (Harita Y, 2008) (Yaoita et al., 2005) (Liu G, 2003) (Dryer SE, 2010) . Linker proteins such as CD2associated protein (CD2AP), and Nck connect the slit diaphragm to the actin skeleton (Coward et al., 2005) (Jones et al., 2009). Also, proteins that are directly part of the slit diaphragm but are involved in regulating the actin cyskeleton include synaptopodin, the GTPase dynamin and cytoplasmic Cathepsin L (CatL) (Sever S, 2007). Nephrin, a member of the immunoglobulin family, is regarded as the critical structural component of the slit diaphragm of podocytes as it is composed of an extracellular and intracellular domain capable of transducing signals between neighboring podocytes, and is the protein that bridges the distance between interdigitating foot processes (Zhu et al., 2008). Encoded by the NPHSI gene, both humans and experimental mice models lacking nephrin or a mutation in the NPHSI gene are born without typical slit diaphragms and exhibit severe podocyte abnormalities and massive proteinuria in utero and at birth (Kuusniemi et al., 2006). The Src family kinase Fyn, controls the phosphorylation of nephrin and when phosphorylated together with CD2AP and podocin, phosphoinositide-3-OH kinase (PI3K)-dependent Akt activation is induced, which is thought to inhibit podocyte apoptosis (Huber et al., 2003). Phosphorylation of nephrin also promotes Nck-dependent actin rearrangements, indicating an important role for nephrin in preventing foot process effacement (Verma et al. 2006). The transmembrane proteins Neph1 and Neph2 are structurally related to nephrin, containing extracellular IgG-like motifs, and interact with nephrin during intracellular signaling. FAT1 and FAT2, like Neph1 and Neph2, are part of the slit diaphragm but contain cadherin-like repeats (Tryggvason et al., 2006). Podocin is a membrane-associated intracellular protein, and is involved in the organization of the slit diaphragm complex, interacting with both nephrin and CD2AP (Schwarz et al., 2001). $\mathrm{CD} 2 \mathrm{AP}$ is linked to nephrin, podocin and the actin cytoskeleton, transducing signals to the actin cytoskeleton. Recently, attention has focused on TRPC6, a calcium channel present in the slit diaphragm complex interacting with both nephrin and podocin, and involved in podocyte mechanosensation and regulation of gene transcription (Moller et al., 2009). Synaptopodin, an actin-binding protein, is present in the foot process and involved in actin elongation through a-actinin-4 by modulating RhoA signaling (Asanuma et al., 2005) (Asanuma et al., 2006). The GTPase dynamin is also involved in actin modulation, and both GTP-bound dynamin and unphosphorylated synaptopodin are direct targets of the proteolytic activity of the cysteine protease Cathepsin L (Reiser et al., 2004). Undoubtedly, an intricate alignment of the slit diaphragm proteins is pivotal for proper filtration and renal function and disruption to the expression or function of these proteins alters the intercellular junctions and cytoskeletal structures of the foot processes and the cell takes on an effaced phenotype, pathologically known as effacement. Thus, foot processes effacement is identified as the hallmark in the development of proteinuric kidney disease (Kwoh et al., 2006) (Perysinaki GS, 2011).

As well as having the role of maintaining glomerular structural and functional integrity, podocytes also play a key role in autocrine signalling. Podocytes are essential suppliers of 
vascular endothelial growth factor A (VEGF-A) to the glomerular capillary endothelium and with glomerular endothelial cells, they are among the few cell types where VEGF-A is expressed throughout life (Tufro et al., 1999) (Simon et al., 1995). In addition, injured podocytes have been reported to upregulate expression of the transmembrane protein B7-1 (or CD80) on their surface, normally expressed on B cells and other antigen-presenting cells (Reiser J, 2004). To illustrate, in response to B7-1 activation due to binding of LPS to toll-like receptor-4 (TLR4), the podocyte slit diaphragm is rearranged leading to proteinuria, a response not seen in B7-1 knockout mice (Reiser J, 2004), suggesting that the podocyte is part of the innate immune system and has a role in danger signaling (Reiser J, 2004) (D'Agati, 2008).

Clearly, the podocyte is a highly complex cell involved in maintaining normal capillary health, other glomerular cells and structures as well as being a gateway for the perm-selectivity of the glomerular filtrate. Given the terminally differentiated nature of podocytes, loss of these cells cannot be compensated by regenerative proliferation. Thus an intricate alignment of the slit diaphragm proteins is pivotal for proper filtration and renal function. Hence, podocytes are key contributors to glomerular disease and understanding the cellular mechanisms governing their response to injury is imperative to the development of therapeutic strategies to directly protect podocytes in disease situations from advancing to kidney sclerosis.

\section{Role of podocytes in glomerular disease}

Glomerular diseases (including diabetic kidney disease) are the most common cause of endstage kidney failure throughout the world. They comprise a variety of heterogenous disorders that are due to acute or chronic injury of the renal glomeruli. Whether they are a primary disease process or a secondary manifestation of a systemic disease process, the working paradigm is that they all converge onto few pathways of disease progression.

Over the last decade, immense knowledge has emerged implicating podocytes as key players in a broad range of glomerular diseases. Injury to the podocytes is the initiating cause of many renal diseases, leading to proteinuria with possible progression to end-stage renal disease (Leeuwis et al., 2010). Human podocytopathies include focal and segmental glomerular sclerosis (FSGS), minimal change disease, membranous nephropathy, collapsing glomerulopathy and diabetic nephropathy and more recently podocytes have also been in implicated in proliferative forms of GN such as crescentic glomerulonephritis (Thorner et al., 2008) (Moeller et al., 2004) . The filtration barrier is the major pathogenic site in almost all glomerular diseases and its study is therefore of clinical significance. When podocytes are injured, as in many forms of glomerulonephritides, the podocytes respond by retracting their foot processes into their cell bodies to form flattened dense epithelium, morphologically designated foot process effacement (Harita et al., 2006) (Mundel and Shankland, 2002). These podocytic changes lead to precipitous protein leakage into the urine, which over a chronic period of time culiminates in the development of glomerulosclerosis and end-stage kidney failure (Smoyer and Mundel, 1998). Thus, the intrinsic defect in the cytoskeletal proteins renders podocytes non-functional as the actin cytoskeletal organisation changes from parallel contractile bundles into a dense network, culminating in foot processes effacement, subsequent proteinuria and sclerosis.

Podocyte contribution is essential to the formation of glomerular crescent as they populate cellular crescents in human and experimental models of CGN (Thorner et al., 2008) (Bariety et al., 2005) (Moeller et al., 2004) (Le Hir et al., 2001). So far, the earliest documentation of 
podocyte response to injury in a model of crescentic glomerulonephritis is from the ultrastructural studies conducted by Le Hir et al., 2001, whereby podocytes formed bridges glomerular tuft area and Bowman's capsule in mice ten days injection with rabbit anti-GBM serum (Le Hir et al., 2001). These findings were the first to provide a link between the initial inflammatory injury processes occurring within the endocapillary compartment of the glomerular tuft and the formation of a multilayered cellular crescent in bowman's space (Le Hir et al., 2001). What causes these presumably terminally differentiated visceral cells to acquire a phenotype favourable for cytoskeletal reorganisation leading to contact with the parietal basement membrane of Bowman's capsule, culminating in the formation of podocyte bridges is yet to be explored.

\section{The mammalian target of rapamycin complex signal transduction pathway}

The coordinated control of cell growth to produce a genetically predetermined cell size, shape, survival and proliferation is greatly influenced by a ubiquitously expressed serine/threonine kinase known as the mammalian target of rapamycin (mTOR). mTOR functions to regulate a variety of cellular activites that are sensitive to environmental stress (Foster, 2007) (Sabatini, 2006). Our current understanding of mTOR dates back to the initial discoverers of its inhibitor, Rapamycin, about three decades ago on the Southern Pacific Ocean island named Easter Island, known as "Rapa Nui" by the language of its native Polynesian inhabitants (Yang and Guan, 2007). The bacterial strain, Streptomyces hygroscopicus, which was first isolated from this island secrete a potent anti-fungal macrolide that was named rapamycin after the location of its discovery (Yang and Guan, 2007). Rapamycin was initially developed as an anti-fungal agent (Loewith, 2011). However, its major application quickly developed after rapamycin was proven to have immunosuppressive and anti-proliferative properties (Yang and Guan 2007). To date, rapamycin (or sirolimus) has become routinely used clinically for immunosuppression in organ transplantation, prevention of restenosis post-angioplasty, and chemotherapy in a variety of cancers (Dobashi et al., 2009) (Yang and Guan, 2007).

mTORC1 Signaling pathway: mTOR is the downstream effector protein belonging to the phosphatidylinositol (PIKK)-related kinase family with a predicted molecular weight of $290 \mathrm{kDa}$, and is part of the PI-3 Kinase pathway which is part of the tyrosine kinase activation cascade (Vogt, 2001). The physiological importance of the primordial mTOR is undoubtedly demonstrated by the fact that the knockout of mTOR in mice is embryonically lethal (Gangloff et al., 2004) (Murakami M, 2004). In mammalian cells, mTOR signaling consist of two distinct multiprotein complexes named mTOR Complex-1 (TOR-raptor-G $\beta \mathrm{L}$ ) and mTOR Complex-2 (TOR-rictor-G $\beta \mathrm{L}$ ), and each complex is evolutionarily conserved from yeast to mammalian eukaryotes with distinct and essential roles in maintenance of cell function and structure (Loewith R, 2002). It is now well established that the raptorcontaining mTOR complex (mTORC1) is rapamycin-sensitive (Sabatini et al., 1994), whereas the rictor-containing mTOR complex (mTORC2) is rapamycin-insensitive (Sarbassov et al., 2006). In contrast to mTORC1, mTORC2 regulates growth factor signalling, cell survival (AKT) and cytoskeletal reorganization (Facchinetti et al., 2008) (Bhaskar PT, 2007) (Jacinto et al., 2004). However, as much of our current understanding of mTOR is limited to mTORC1, because of the availability and wide use of rapamycin. The factors promoting mTORC2 activation are not well defined and may be controlled by the activation states of multiple mTORC1 effector proteins (Gossage L, 2010). 


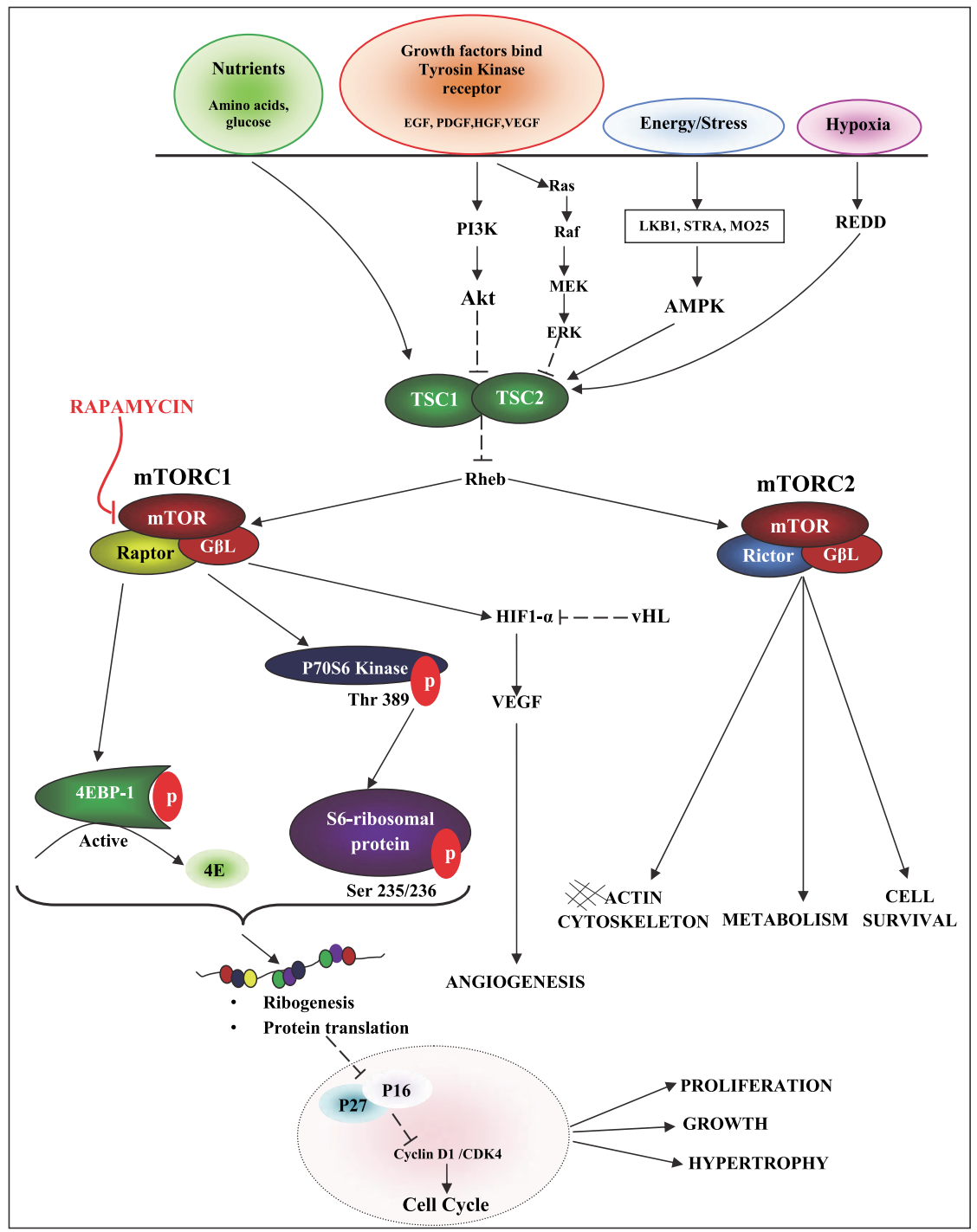

Fig. 1. Mammalian target of rapamycin (mTOR) signaling pathway. mTOR incorporates growth factor activation from the tyrosine receptor kinase to control several catabolic and anabolic processes that collectively determine cell growth and metabolism. mTOR is an atypical serine/threonine kinases. In response to growth factors, Akt phosphorylates and inactivates TSC1-TSC2, allowing Rheb to activate mTOR. Low energy (cellular energy status) inhibits mTORC by activating AMPK, which phosphorylates and activates TSC1-TSC2. mTOR controls protein synthesis by phosphorylating and inactivating the translational inhibitor 4EBP-1 and by phosphorylating and activating P70S6 kinase. mTORC2 promotes cell survival through direct phosphorylation of serine 473 in the hydrophobic motif of AKT. The immunosuppressant rapamycin in complex with FKBP inhibits mTOR when bound to raptor. 
Activities of mTORC1: The mTORC1 arm of mTOR signaling pathway promotes cell cycle progression through $\mathrm{G}_{1}$-phase and proliferation through multiple molecular mechanisms. mTORC1 signaling functions via the two earliest recognized and best-characterised downstream effector proteins namely, SK1 (p70 ribosomal protein S6 kinase 1) and eukaryotic initiation factor (eIF) 4E-binding protein 1 (4EBP1) that are involved in the regulated control of protein synthesis/ribosome biogenesis and cap-dependent translation initiation (Yang and Guan 2007). The pathway is activated as follows: Firstly, mTOR incorporates growth factor activation from the tyrosine kinase receptor to activate AKT via phosphorylation at the threonine-308 residue site, which results in phosphorylation and subsequent inactivation of the endogenous mTOR complexes inhibitors, the tuberous sclerosis complexes-1 and 2 (TSC1-TSC2). Upon phosphorylation of TSC1-TSC2 heterodimer, Rheb is released which positively regulates mTORC1 for activation via phosphorylation at the serine 2448 site (Yang and Guan 2007). The activation of mTORC1 results in increased protein synthesis by phosphorylating and inactivating the translational inhibitor 4E-BP and by phosphorylating and activating S6 kinase (S6K). S6K, in addition to phosphorylating various translational targets. It also phosphorylates and inhibits insulin receptor substrate 1 (IRS-1) as part of a negative feedback loop that attenuates insulin signaling. mTORC2 promotes cell survival through direct phosphorylation of serine 473 in the hydrophobic motif of AKT. The immunosuppressant rapamycin in complex with FKBP binds and inhibits mTOR when bound to raptor. Activation of the protein kinase p70 S6 kinase (p70s6k) and subsequent phosphorylation of $40 \mathrm{~S}$ subunit of the S6 ribosomal protein regulates the translation of ribosome proteins and components of the translational machinery. mTORC1 also phosphorylates 4E-BP1, which releases it from the translation factor, eIF-4E, and allows the latter to initiate translation of messenger RNAs. TORC1 also regulates proliferation through modulation of cell cycle regulatory proteins, involving the cyclin D1/E-cyclin-dependent kinase (Cdk)-2/4-retinoblastoma pathway (Berthet and Kaldis, 2007). In particular, eIF4E regulates the translation of the Cyclin D1 and D3 genes, which leads to reduced formation of cyclinD3-cdk4 kinase (Hleb M, 2004). TORC1 activation is correlated with the protein expression of cdk2 (Chen et al., 2009) and also increases the expression of p27kip1 (the endogenous inhibitor of cdks 2 and 4), through undefined mechanisms (Sun et al., 2008) (Wang et al., 2001). mTORC1 is also a negative regulator of autophagy (one of the major systems which regulate cellular protein degradation) (Meijer and Codogno, 2004).

The earliest recognized and best-studied mTORC1 downstream effector proteins are SK1 (p70 ribosomal protein S6 kinase 1) and eukaryotic initiation factor (eIF) 4E-binding protein 1 (4EBP1). Under basal conditions, S6KI and 4EBP1 are bound to eIF3 and remain inactive (Holz MK, 2005) mTOR-dependent phosphorylation of 4EBP1 releases eIF4E, initiating capdependent de novo translation of various species of mRNA (Brunn et al., 1997 and Gingras et al., 2004). Another set of downstream effectors of mTOR are the serine/threonine protein kinases, S6 kinase 1 (S6K1) and S6 kinase 2 (S6K2), both of which are responsible for phosphorylation of the 40S ribosomal protein S6 (rpS6) (Murakami et al., 2004, Sarbassov et al., 2004 and Shima et al., 1998). mTOR activation of S6K results in phosphorylation of S6 ribosomal protein, leading to recruitment of the $40 \mathrm{~S}$ ribosome subunit and translation of 5'terminal oligopyrimidine tract (5'TOP) mRNA (Jefferies et al., 1997). These 5'TOP mRNA encode primarily ribosomal proteins and other components of the translational apparatus (Meyuhas et al., 2000). Thus, by controlling S6K activity, mTOR also regulates the abundance of translational machinery. Under favorable growth conditions, mTOR is 
activated and S6K and 4E-BP1 are phosphorylated, leading to new RNA and protein synthesis. Proliferation of intrinsic glomerular cells, characterized by increased RNA and protein synthesis with DNA replication and cell division is fundamental in crescent formation in crescentic glomerulonephritis. It may well be that mTOR plays a critical role in this disease process. Thus, the judicious attenuation of proliferation with mTOR inhibitors may potentially provide an option to limiting the progression of renal injury from progressing to crescentic glomerulonephritis.

\section{Implication of mTOR signaling in renal disease}

The availability of the mTOR inhibitor $\left(\mathrm{mTOR}_{\mathrm{i}}\right)$ rapamycin, which is used clinically as an immunosuppressant drug to prevent rejection in organ transplantation, has helped to identify a large signaling network around mTOR signaling leading to extensive studies for its effect on cell proliferation and apoptosis not just in cancer but in both human and experimental in vivo and in vitro models of glomerular disease. Nevertheless, whilst some studies suggest mTOR inhibition by rapamycin might halt or reverse glomerulopathies (Mori H, 2009) (Inoki, 2008) (Lloberas et al., 2006) (Sakaguchi et al., 2006) (Rangan and Coombes, 2007) other studies documented an increase in proteinuria and glomerulosclerosis in patients and in animal models following rapamycin treatment (Torras J, 2009) (Amer and Cosio, 2009) (Munivenkatappa et al., 2010) (Letavernier et al., 2007), suggesting a significance for mTORC1 signaling in endogenous and compensatory regenerative repair of renal cells in glomerulopathies. To add, most studies regarding the role of mTOR signaling in renal disease are based on pharmacological intervention with rapamycin (and as mTORC1 is expressed in resident as well as infiltrating cells) this approach does not allow for distinguishing the specific role of blocking mTORC1 in intrinsic renal cells. Furthermore, off-target effects have been described with long-standing application of rapamycin, most notably the inhibition of mTORC2 (Sarbassov et al., 2006). Therefore, tissue-specific analysis of mTOR signaling is required for an in-depth understanding of the functional and cell autonomous role of mTOR in diabetic nephropathy and other glomerular diseases.

\section{Role of TORC1 in normal podocyte biology}

The mTOR signalling pathway has emerged as having an important role in maintaining podocyte survival and cytoskeletal structure. Recently, elegant studies by Godel et al. and Inoki et al., conducted in conditional knockout mice, have comprehensively verified the critical role that TORC1 plays in maintaining normal podocyte function and glomerular architecture. Godel et al. showed that podocyte-specific deletion of TORC1 (Raptorflox/flox) in conditional knockout mice caused heavy proteinuria at 8 weeks of age, associated with development of segmental glomerulsclerosis (particulary in juxtamedullary glomeruli), reduced expression of slit diaphragm proteins (nephrin, podocin and Par3), foot process effacement and increased mortality after 8 months of age. The dependence of normal podocyte biology on TORC1 was also determined by genetic background (with conditional TORC1 deletion in ICR mice being more prone to proteinuria than those from a C57BL/6 background) as well as age (suppression of TORC1 was more resistant in causing proteinuria in adult mice). Godel and co-authors also demonstrated that the combined inactivation of TORC1 and TORC2 (using Raptor/Rictorpodocyte conditional mice) caused a rapid onset of nephrotic syndrome, FSGS, renal failure and death by 8 weeks of age. 
Using an alternative approach, Inoki et al. examined the effects of over-activation of TORC1 in podocytes using podocyte-specific deletion of TSC1 (a negative regulator of TORC1 activation). These studies showed that high TORC1 activation led to mesangial expansion and glomerulosclerosis, proteinuria, podocyte body enlargement and foot process effacement and a reduction in podocyte number (partly due to reduced podocyte attachment) and death due to renal failure by 14 weeks. The molecular and cellular changes underlying these morphological changes included mislocalization of nephrin, cellular dedifferentiation and conversion to a fibroblastic phenotype (as determined by increased expression of desmin and redistribution of zonula occluden-1) and promoted endoplasmic reticulum stress. These effects were rapamycin-sensitive and reversible if attenuated at an early stage, but not after prolonged over-activation. Taken together, the studies by Godel et al. and Inoki et al. highlight that glomerular TORC1 activity in podocytes must kept under low finely regulated levels, with both complete suppression as well as over-expression of TORC1 having deleterious effects on podocyte structure and function.

The molecular mechanisms by which TORC1 regulates normal podocyte function is not yet fully understood. Previous in vitro studies investigating the effects of TORC1 inhibition with sirolimus in cultured podocytes have shown that the TORC1 regulates at least three physiological functions which are essential for normal podocyte surivial as well as functioning of the glomerular filtration barrier: (i) podocytes produce VEGF (an important angiogenic peptide that maintains the normal integrity of the glomerular-endothelial barrier (Eremina, Baelde, \& Quaggin, 2007), in a TORC1 dependent manner; (ii) TORC1 may regulate pro-survival pathways in podocytes. At present, there is only indirect evidence to support this hypothesis. Nephrin and CD2-associated protein (CD2AP) constitutively regulate PI3K and Akt, both of which are upstream of TORC1 (Huber et al., 2003). Nephrin is a transmembrane protein expressed in podocytes and is linked to the cytoskeleton by CD2AP (Pavenstadt, Kriz, \& Kretzler, 2003). Both are critical factors that maintain the normal function of the slit diaphragm of the podocytes and the glomerular filtration barrier (Pavenstadt et al., 2003). Given that mutations in the genes encoding nephrin or CD2AP lead to FSGS, these studies raise the possibility that their downstream pathways involve TORC1 (Kwoh, Shannon, Miner, \& Shaw, 2006). This postulate has recently been confirmed in two studies showing that sirolimus reduced Akt phosphorylation in cultured podocytes (Letavernier et al., 2008; Vollenbroeker et al., 2008). Akt is a cytoplasmic serine/threonine kinase that regulates programmed cell death by phosphorylating substrates that regulate apoptosis (such as caspase-9, XIAP, MM2); (iii) Lastly, sirolimus altered the cytoskeletal structure of podocytes in vitro. The latter may be due to inhibitory effects on Akt, TORC2 and Wt1(Letavernier et al., 2008; Vollenbroeker et al., 2008). In vitro, podocytes exposed to sirolimus $(20 \mathrm{ng} / \mathrm{ml})$ for 48 hours developed a more roundshaped morphology with reorganization of cytoskeleton and redistribution of actin filaments and focal adhesion proteins (viniculin) to the periphery of the cell (Letavernier et al., 2008; Vollenbroeker et al., 2008). These effects may lead to reduced cell adhesion and migration (Vollenbroeker et al., 2008).

\section{Role of TORC1 in podocytes in human and animal models of glomerular disease}

Glomerular diseases can be classified according to changes in podocyte number and differentiation, as proposed by Shankland and colleagues (Griffin et al. 2003)(Figure 2). Podocyte number is normal or decreased in minimal change disease, focal segmental 
glomerulosclerosis and diabetic kidney disease, whereas it is increased with an dedifferentiated phenotype in HIV nephropathy, collapsing variant of focal segmental glomerulosclerosis and crescentic glomerulonephritis. Loss of Podocytes (due to cell death and detachment from the glomerular basement membrane) is a key determinant of progression to kidney failure, regardless of the cause. Our knowledge about the role of TORC1 in podocytes in glomerular disease is incomplete and is summarised and discussed below.

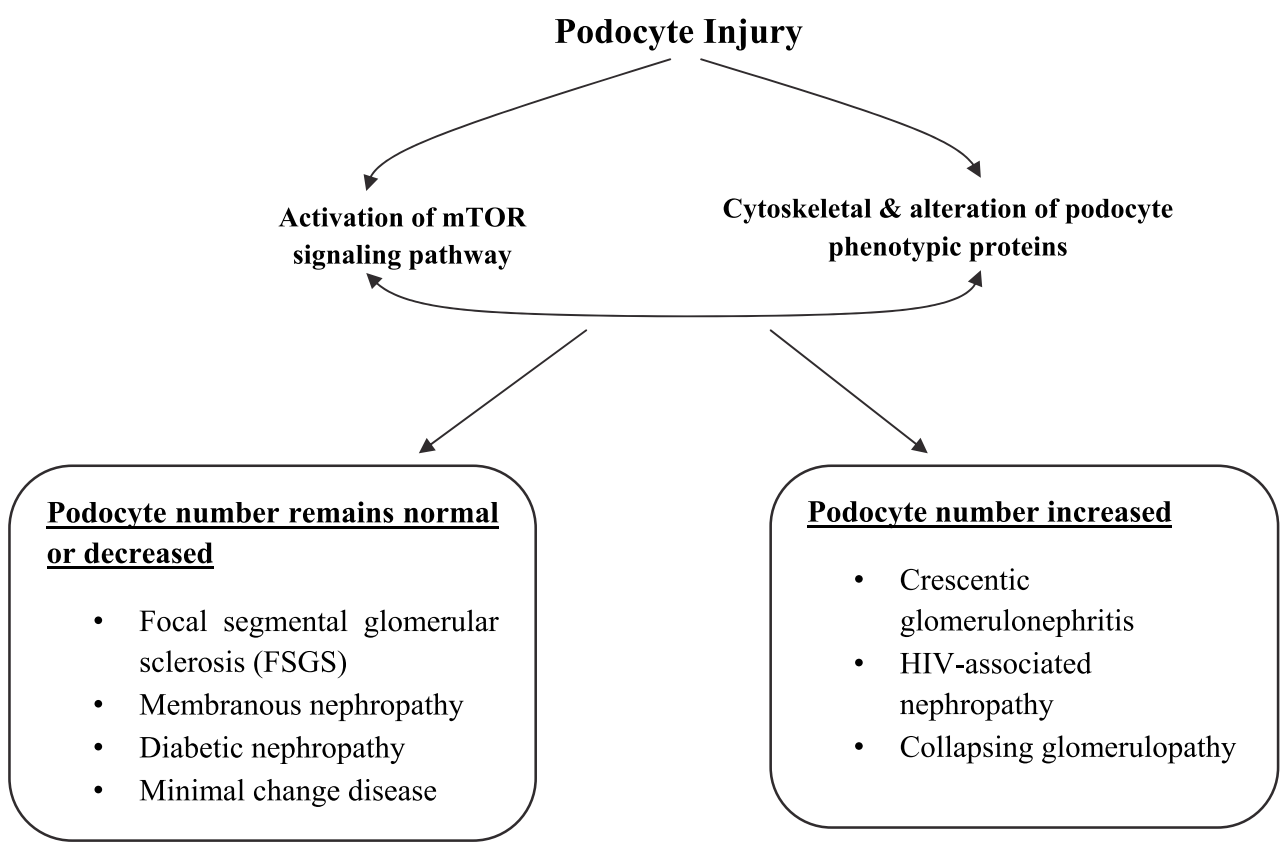

Fig. 2. Classification of glomerular diseases according to changes in podocyte number and differentiation, as proposed by Shankland and colleagues (Griffin et al. 2006).

\subsection{Glomerular diseases in which podocyte number decreased or normal}

Minimal change disease/focal segmental glomerulosclerosis. Several studies have examined the effects of sirolimus in animal models of glomerular diseases associated with heavy proteinuria and reduced or normal podocyte number. These models resemble the human counterparts of FSGS and minimal change disease. Both everolimus and sirolimus were renoprotective when administered during the early stages of non-immune FSGS induced by either puromycin (Daniel et al., 2000) or adriamycin (Rangan and Coombes, 2007) in rats. In adriamycin nephropathy, commencement of sirolimus during the established, but early phase of disease (day 14 to 49), attenuated the increase in kidney weight, glomerular capillary tuft enlargement, glomerulosclerosis and tubulointerstitial fibrosis as well as renal cortical TGF- $\beta$ (Rangan and Coombes, 2007), whereas interstitial inflammation and peritubular myofibroblast accumulation were unaffected. Interestingly, the examination of sirolimus on proteinuria and subsequent podocyte damage in two contrasting renal disease models, with clearly different pathophysiological mechanisms (a glomerular toxico- 
immunological model induced by puromycin aminonucleoside, and a chronic hyperfiltration and inflammatory model by mass reduction), both treated with a fixed high sirolimus dose revealed, significant increases in proteinuria, together with a significant fall in podocin immunofluorescence, as well as clear additional damage to podocyte foot processes (Torras et al., 2009). Conversely, after mass reduction, rapamycin produced lower levels of proteinuria and amelioration of inflammatory and pro-fibrotic damage. In contrast to the puromycin model, higher glomerular podocin and nephrin expression and amelioration of glomerular ultrastructural damage were found (Torras et al., 2009). Thus these studies suggest sirolimus has dual opposing effects, with proteinuria and podocyte damage aggravation in the glomerular model and a nephro-protective effect in the chronic inflammatory tubulointerstitial model. To add, sirolimus has also been reported to damage podocytes in rats with protein overload nephropathy (Cia et al., 2011). Collectively these findings indicate that sirolimus produces slight alterations in podocyte structure when acting on healthy podocytes, but it clearly worsens those podocytes damaged by other concomitant injury.

In humans with FSGS, the role of TORC1 in podocytes in disease pathogenesis has not been directly evaluated. Clinical trial evidence however suggests that TORC1 has a dichotomous role (congruent with data from TORC1-conditional knockout mice) indicating that its role in disease is finely balanced. (Pollak, 2008, Fervenza et al., 2004, Tumlin et al., 2006, Cho et al., 2007, Aymanns et al., 2006). In a prospective open label trial, conducted by Tumlin and colleagues (Tumlin et al., 2006), sirolimus induced complete remission (defined as $<300 \mathrm{mg}$ protein/24h after 6 months) in 4 of the 21 patients, and partial remission (defined as a $50 \%$ reduction of baseline proteinuria) in 8 of the 21 patients tested within the 6 month period. Twelve of the responsive patients, along with 2 others who saw a $30 \%$ reduction in proteinuria continued sirolimus therapy for another 6 months. In these patients, baseline proteinuria fell significantly within the first 6 months, however this decline in proteinuria became statistically insignificant from the period of 6 months to 12 months. Overall, the study found that sirolimus was able to stabilize the renal function of patients with FSGS, by not only reducing proteinuria, but also glomerular pore size and increasing glomerular ultrafiltration coefficient (Fervenza et al., 2004). On the other hand, Patel and colleagues (Patel et al., 2005) reported that a combination of tacrolimus with sirolimus was beneficial in reducing proteinuria and inducing remission in a patient with a long history of refractory minimal change nephropathy. In contrast, other smaller pilot studies in humans highlighted the possible nephrotoxic effects of sirolimus in patients with FSGS (Tumlin et al., 2006, Cho et al., 2007). A common observation amongst all the studies was that a longer duration of disease was associated with resistance to sirolimus therapy and a risk for renal dysfunction. Therefore, genetic factors, stage of disease and level of TORC1 inhibition may be factors underlying these variable results in humans.

Membranous nephropathy. The effects of sirolimus have been examined in both the passive and active Heymann nephritis model of membranous nephropathy (Cavallo, 1994), but the specific effects on podocytes has not been assessed. In a nephrectomized model of passive Heyman nephritis, sirolimus reduced the expression of pro-inflammatory and pro-fibrotic genes, tubulointerstitial inflammation and fibrosis as well as compensatory renal hypertrophy (Bonegio et al., 2005). In the active model, sirolimus exhibited protective effects during both the induction as well as the chronic phases (Naumovic et al., 2007). The efficacy 
of sirolimus in human membranous nephropathy is currently being evaluated in an NIHsponsored clinical trial.

Diabetic kidney disease. Sirolimus is known to prevent the development of diabetes in nonobese diabetic mouse, which is an autoimmune model of insulin-dependent diabetes, presumably due to immunomodulatory effects (Baeder et al., 1992). In the kidney, the expression of mTORC1 is upregulated in animals with diabetes, possibly through an AMPK-dependent manner (Lee et al., 2007). In addition, several groups have investigated the role of sirolimus and mTORC1 in mediating renal injury associated with diabetes. These studies showed that sirolimus reduced renal hypertrophy associated with hyperglycaemia and that it also has some anti-inflammatory and anti-fibrotic effects (Yang et al., 2007, Sataranatarajan et al., 2007, Lee et al., 2007, Sakaguchi et al., 2006, Lloberas et al., 2006, Nagai et al., 2005). For example, the treatment of rats with streptozotocin (STZ)-induced diabetes with sirolimus from week 16 to 20 reduced albuminuria, phosphorylated Akt and mTORC1 expression and growth factor expression of TGF- $\beta 1$ (Lloberas et al., 2006). These effects were comparable to diabetic animals treated with insulin (Lloberas et al., 2006). Sirolimus had similar effects in $\mathrm{db} / \mathrm{db}$ mice with diabetes (Sataranatarajan et al., 2007). The effects of sirolimus in human diabetic nephropathy have not been directly evaluated. However, Senior and colleagues reported in a retrospective case series, 3 out 62 clinical islet transplant recipients developed proteinuria when converted from CNI-based regimen to a CNI-free/sirolimus-based treatment (Senior et al., 2005). At least two of the three patients had underlying established diabetic nephropathy, which progressed following islet transplantation. Another five patients developed microalbuminuria. The proteinuria improved with increased doses of $\mathrm{CNI}$, suggesting that the mechanisms of proteinuria may be similar to that described for patients with chronic allograft nephropathy (CAN) (i.e. partly due to CNI withdrawal) (Senior et al., 2005).

Chronic kidney disease. In the remnant kidney model in rats and mice, $5 / 6^{\text {th }}$ of the kidneys are removed either by nephrectomy or infarction through the selective ligation of the renal arteries. This model recapitulates some of the features associated with chronic kidney progression in humans. In this model, divergent effects of mTOR inhibitors have been observed, but this may depend on the timing of their administration, similar to what was observed in the glomerular disease models described earlier (Diekmann et al., 2007, Vogelbacher et al., 2007). For example, Vogelbacher and co-workers found that starting everolimus three days after induction of the model, suppressed glomerular endothelial and mesangial cell proliferation, and worsened structural renal injury and proteinuria (Vogelbacher et al., 2007). In contrast, when sirolimus was started at week 6, it improved proteinuria, fibrosis and inflammation (Diekmann et al., 2007). Sirolimus attenuated glomerular VEGF production in both studies and paradoxically increased serum levels of VEGF (Diekmann et al., 2007).

\subsection{Glomerular diseases in which podocyte number increased}

Crescentic glomerulonephritis. mTORC1 may mediate both the immunopathogenesis as well as the local glomerular cell proliferation that occurs in crescentic glomerulonephritis. For example, in mice the overexpression of HIF-1a (Ding et al., 2006). Two recent studies have evaluated the effects of sirolimus in murine models of crescentic glomerulonephritis. In 
mice, prophylactic treatment with sirolimus, 5 days prior to disease induction, decreased the proliferation of inflammatory cells and autologous antibody production, but did not alter the lymph node expression of CD4+CD25-FoxP3 regulatory cells was detected (Hochegger et al., 2008). In contrast, when sirolimus was commenced on day 14 of established disease, deleterious effects occurred, including an increase in proteinuria and worsening of inflammatory cell infiltration (Hochegger et al., 2008). In rats with crescentic glomerulonephritis induced by nephrotoxic serum, treatment with sirolimus during the early stage (1 day after immunization) reduced glomerular cell proliferation, tubulointerstitial damage and fibrinoid necrosis, as well as increasing urine volume and preventing the decline in renal function (Succar et al., 2007). Of note, the degree of histopathological protection following sirolimus treatment was much greater in the tubulointerstitial compartment than in the glomerulus, and was associated with reductions in both the glomerular and tubulointerstitial phosphorylation of the S6 ribosomal protein (Succar et al., 2007).

HIV-nephropathy. The most common, or "classical", type of HIV-associated nephropathy (HIVAN) is a collapsing FSGS, though other forms of kidney disease may also occur with HIV (Medapalli, 2011) (Kimmel et al., 2003). Both glomerular and tubular lesions are characterized by a proliferative phenotype in HIVAN, and mTORC1 pathway was been implicated to contribute to the development of the HIVAN phenotype, as demonstrated in Kumar et al., 2010, whereby, renal tissues of transgenic mice showed enhanced phosphorylation of p70S6 kinase, 4EBP1 and eIF4B, HIF1- $\alpha$, VEGF and an associated diminished phosphorylation of eEF2 (Kumar D, 2010). Here transgenic mice receiving rapamycin not only showed inhibition of the mTOR-associated downstream signaling but also displayed attenuated renal lesions suggesting that the mTOR pathway contributes to the HIVAN phenotype and that inhibition of the mTOR pathway can be used as a therapeutic strategy to alter the course of HIVAN (Kumar D, 2010).

\section{Therapeutic implications of targeting TORC1 in podocytes in therapy of human glomerular diseases}

The foregoing data clearly support that the TORC pathway has an important role in the pathogenesis of glomerular disease. However, our knowledge in human disease is in its infancy and there remain several significant challenges in translating the known role of TORC in podocytes to the therapy of human glomerular disease. Firstly, further studies are needed to confirm the expression and functional role of TORC in human disease. Biopsy studies of the localization of TORC pathway in glomerular disease are essential. Furthermore, serial assessment of biomarkers indication TORC activity will allow the level of expression to be determined according to stage of disease. Such assessment may include the expression of TORC in podocytes of the urine. Second, further refinements in the way in which TORC is modulated in vivo are required. This may require evaluation of low-dose sirolimus in conjunction with assessment of biomarkers that modulate the bioactivity of TORC; the development of more specific drug-inhibitors that target the TORC pathway as well as more specific methods of drug-delivery that target the podocyte. In essence, further data in humans is currently lacking and currently essential for furthering the role of TORC modulation in podocytes. 


\section{Summary and conclusions}

Podocytes form the final barrier to protein loss, which explains why podocyte injury is typically associated with marked proteinuria. Indeed, all forms of glomerular disease are characterized by some degree of podocyte abnormality. Recent advancement in the understanding of the role of mTOR signalling pathway have emphasized the important role this key cell signalling transduction pathway plays in podocyte function both in normal and disease conditions. However, much more work is still needed to understand how mTOR signalling in podocytes can be modulated to allow the effective therapy of human glomerular disease.

\section{References}

Akilesh S, H. T., Wu H, Wang G, Hartleben B, Kopp JB, Miner JH, Roopenian DC, Unanue ER, Shaw AS 2008. Podocytes use FcRn to clear IgG from the glomerular basement membrane. Proc Natl Acad Sci USA, 105, 967-972.

Amer, H. \& Cosio, F. G. 2009. Significance and management of proteinuria in kidney transplant recipients. J Am Soc Nephrol, 20, 2490-2.

Asanuma, K., Kim, K., Oh, J., Giardino, L., Chabanis, S., Faul, C., Reiser, J. \& Mundel, P. 2005. Synaptopodin regulates the actin-bundling activity of alpha-actinin in an isoform-specific manner. J Clin Invest, 115, 1188-98.

Asanuma, K., Yanagida-Asanuma, E., Faul, C., Tomino, Y., Kim, K. \& Mundel, P. 2006. Synaptopodin orchestrates actin organization and cell motility via regulation of RhoA signalling. Nat Cell Biol, 8, 485-91.

Aymanns, C., Voehringer, M., Rasche, F. M. \& Keller, F. 2006. Effects of sirolimus on proteinuria and renal function in focal segmental glomerulonephritis. Int $\mathrm{J}$ Clin Pharmacol Ther, 44, 675-6.

Baeder, W. L., Sredy, J., Sehgal, S. N., Chang, J. Y. \& Adams, L. M. 1992. Rapamycin prevents the onset of insulin-dependent diabetes mellitus (IDDM) in NOD mice. Clin Exp Immunol, 89, 174-8.

Bariety, J., Bruneval, P., Meyrier, A., Mandet, C., Hill, G. \& Jacquot, C. 2005. Podocyte involvement in human immune crescentic glomerulonephritis. Kidney Int, 68, 110919.

Benzing, T. 2004. Signaling at the slit diaphragm. J Am Soc Nephrol, 15, 1382-1391.

Berthet, C. \& Kaldis, P. 2007. Cell-specific responses to loss of cyclin-dependent kinases. Oncogene, 26, 4469-77.

Bhaskar PT, H. N. 2007. The two TORCs and Akt. Dev Cell, 12, 487-502.

Bonegio, R. G., Fuhro, R., Wang, Z., Valeri, C. R., Andry, C., Salant, D. J. \& Lieberthal, W. 2005. Rapamycin ameliorates proteinuria-associated tubulointerstitial inflammation and fibrosis in experimental membranous nephropathy. J Am Soc Nephrol, 16, 206372.

Burt, D., Salvidio, G., Tarabra, E., Barutta, F., Pinach, S., Dentelli, P., Camussi, G., Perin, P. C. \& Gruden, G. 2007. The monocyte chemoattractant protein-1/cognate CC chemokine receptor 2 system affects cell motility in cultured human podocytes. Am J Pathol, 171, 1789-99. 
Cavallo, T. 1994. Membranous nephropathy. Insights from Heymann nephritis. Am J Pathol, 144, 651-8.

Chen, S. J., Nakahara, T., Takahara, M., Kido, M., Dugu, L., Uchi, H., Takeuchi, S., Tu, Y. T., Moroi, Y. \& Furue, M. 2009. Activation of the mammalian target of rapamycin signalling pathway in epidermal tumours and its correlation with cyclin-dependent kinase 2. Br J Dermatol, 160, 442-5.

Cho, M. E., Hurley, J. K. \& Kopp, J. B. 2007. Sirolimus therapy of focal segmental glomerulosclerosis is associated with nephrotoxicity. Am J Kidney Dis, 49, 310-7.

Coward, R. J., Foster, R. R., Patton, D., Ni, L., Lennon, R., Bates, D. O., Harper, S. J., Mathieson, P. W. \& Saleem, M. A. 2005. Nephrotic plasma alters slit diaphragmdependent signaling and translocates nephrin, Podocin, and CD2 associated protein in cultured human podocytes. J Am Soc Nephrol, 16, 629-37.

D'agati, V. D. 2008. Podocyte injury in focal segmental glomerulosclerosis: Lessons from animal models (a play in five acts). Kidney Int, 73, 399-406.

Daniel, C., Ziswiler, R., Frey, B., Pfister, M. \& Marti, H. P. 2000. Proinflammatory effects in experimental mesangial proliferative glomerulonephritis of the immunosuppressive agent SDZ RAD, a rapamycin derivative. Exp Nephrol, 8, 52-62.

Diekmann, F., Rovira, J., Carreras, J., Arellano, E. M., Banon-Maneus, E., Ramirez-Bajo, M. J., Gutierrez-Dalmau, A., Brunet, M. \& Campistol, J. M. 2007. Mammalian target of rapamycin inhibition halts the progression of proteinuria in a rat model of reduced renal mass. J Am Soc Nephrol, 18, 2653-60.

Ding, M., Cui, S., Li, C., Jothy, S., Haase, V., Steer, B. M., Marsden, P. A., Pippin, J., Shankland, S., Rastaldi, M. P., Cohen, C. D., Kretzler, M. \& Quaggin, S. E. 2006. Loss of the tumor suppressor Vhlh leads to upregulation of Cxcr4 and rapidly progressive glomerulonephritis in mice. Nat Med, 12, 1081-7.

Dobashi, Y., Suzuki, S., Sato, E., Hamada, Y., Yanagawa, T. \& Ooi, A. 2009. EGFR-dependent and independent activation of Akt/mTOR cascade in bone and soft tissue tumors. Mod Pathol, 22, 1328-40.

Dryer SE, R. J. 2010. TRPC6 channels and their binding partners in podocytes: role in glomerular filtration and pathophysiology. Review. Am J Physiol Renal Physiol 299, 689-701

Eremina, V. \& Quaggin, S. E. 2004. The role of VEGF-A in glomerular development and function. Curr Opin Nephrol Hypertens, 13, 9-15.

Eyre J, I. K., Grubb BD, Saleem MA, Mathieson PW, Brunskill NJ, Christensen EI, Topham PS 2007. Statin-sensitive endocytosis of albumin by glomerular podocytes. Am J Physiol Renal Physiol, 292, 674-681

Facchinetti, V., Ouyang, W., Wei, H., Soto, N., Lazorchak, A., Gould, C., Lowry, C., Newton, A. C., Mao, Y., Miao, R. Q., Sessa, W. C., Qin, J., Zhang, P., SU, B. \& Jacinto, E. 2008. The mammalian target of rapamycin complex 2 controls folding and stability of Akt and protein kinase C. Embo J, 27, 1932-43.

Faul, C., Asanuma, K., Yanagida-Asanuma, E., Kim, K. \& Mundel, P. 2007. Actin up: regulation of podocyte structure and function by components of the actin cytoskeleton. Trends Cell Biol, 17, 428-37. 
Fervenza, F. C., Fitzpatrick, P. M., Mertz, J., Erickson, S. B., Liggett, S., Popham, S., Wochos, D. N., Synhavsky, A., Hippler, S., Larson, T. S., Bagniewski, S. M. \& Velosa, J. A. 2004. Acute rapamycin nephrotoxicity in native kidneys of patients with chronic glomerulopathies. Nephrol Dial Transplant, 19, 1288-92.

Foster, D. A. 2007. Regulation of mTOR by phosphatidic acid? Cancer Res, 67, 1-4.

Gangloff, Y. G., Mueller, M., Dann, S. G., Svoboda, P., Sticker, M., Spetz, J. F., Um, S. H., Brown, E. J., Cereghini, S., Thomas, G. \& Kozma, S. C. 2004. Disruption of the mouse mTOR gene leads to early postimplantation lethality and prohibits embryonic stem cell development. Mol Cell Biol, 24, 9508-16.

Gossage L, E. T. 2010. Targeting multiple kinase pathways: a change in paradigm. Clin Cancer Res, 16, 1973-1978.

Griffin, S. V., Petermann, A. T., Durvasula, R. V. \& Shankland, S. J. 2003. Podocyte proliferation and differentiation in glomerular disease: role of cell-cycle regulatory proteins. Nephrol Dial Transplant, 18 Suppl 6, vi8-13.

Guan, F., Villegas, G., Teichman, J., Mundel, P. \& Tufro, A. 2006. Autocrine VEGF-A system in podocytes regulates podocin and its interaction with CD2AP. Am J Physiol Renal Physiol, 291, F422-8.

Harita Y, K. H., Kosako H, Tezuka T, Sekine T, Igarashi T, Hattori S 2008. Neph1, a component of the kidney slit diaphragm, is tyrosine-phosphorylated by the Src family tyrosine kinase and modulates intracellular signaling by binding to Grb2. J Biol Chem 283, 9177-9186.

Harita, Y., Miyauchi, N., Karasawa, T., Suzuki, K., Han, G. D., Koike, H., Igarashi, T., Shimizu, F. \& Kawachi, H. 2006. Altered expression of junctional adhesion molecule 4 in injured podocytes. Am J Physiol Renal Physiol, 290, F335-44.

Hleb M, M. S., Wagner EF, Hanna NN, Sharma N, Park J, Li XC, Strom TB, Padbury JF, Tseng YT, Sharma S 2004. Evidence for cyclin D3 as a novel target of rapamycin in human T lymphocytes. J Biol Chem, 279, 31948-31955.

Hochegger, K., Jansky, G. L., Soleiman, A., Wolf, A. M., Tagwerker, A., Seger, C., Griesmacher, A., Mayer, G. \& Rosenkranz, A. R. 2008. Differential effects of rapamycin in anti-GBM glomerulonephritis. J Am Soc Nephrol, 19, 1520-9.

Holz MK, B. B., Gygi SP, Blenis J 2005. mTOR and S6K1 mediate assembly of the translation preinitiation complex through dynamic protein interchange and ordered phosphorylation events. Cell, 123, 569-580.

Huber, T. B., Hartleben, B., Kim, J., Schmidts, M., Schermer, B., Keil, A., Egger, L., Lecha, R. L., Borner, C., Pavenstadt, H., Shaw, A. S., Walz, G. \& Benzing, T. 2003. Nephrin and CD2AP associate with phosphoinositide 3-OH kinase and stimulate AKTdependent signaling. Mol Cell Biol, 23, 4917-28.

Inoki, K. 2008. Role of TSC-mTOR pathway in diabetic nephropathy. Diabetes Res Clin Pract, 82 Suppl 1, S59-62.

Jacinto, E., Loewith, R., Schmidt, A., Lin, S., Ruegg, M. A., Hall, A. \& Hall, M. N. 2004. Mammalian TOR complex 2 controls the actin cytoskeleton and is rapamycin insensitive. Nat Cell Biol, 6, 1122-8. 
Jones, N., New, L. A., Fortino, M. A., Eremina, V., Ruston, J., Blasutig, I. M., Aoudjit, L., Zou, Y., Liu, X., Yu, G. L., Takano, T., Quaggin, S. E. \& Pawson, T. 2009. Nck proteins maintain the adult glomerular filtration barrier. J Am Soc Nephrol, 20, 1533-43.

Kerjaschki D, S. D., Farquhar MG 1984. Identification and characterization of podocalyxin-the major sialoprotein of the renal glomerular epithelial cell. J Cell Biol, 98, 15911596.

Kimmel, P. L., Barisoni, L. \& Kopp, J. B. 2003. Pathogenesis and treatment of HIV-associated renal diseases: lessons from clinical and animal studies, molecular pathologic correlations, and genetic investigations. Ann Intern Med, 139, 214-26.

Koop, K., Eikmans, M., Wehland, M., Baelde, H., Ijpelaar, D., Kreutz, R., Kawachi, H., Kerjaschki, D., De Heer, E. \& Bruijn, J. A. 2008. Selective loss of podoplanin protein expression accompanies proteinuria and precedes alterations in podocyte morphology in a spontaneous proteinuric rat model. Am J Pathol, 173, 315-26.

Kumar D, K. S., Yadav A, Sataranatarajan K, Kasinath BS, Chander PN, Singhal PC 2010. HIV-associated nephropathy: role of mammalian target of rapamycin pathway. Am J Pathol, 177, 813-821.

Kuusniemi, A. M., Merenmies, J., Lahdenkari, A. T., Holmberg, C., Salmela, K., Karikoski, R., Rapola, J. \& Jalanko, H. 2006. Glomerular sclerosis in kidneys with congenital nephrotic syndrome (NPHS1). Kidney Int, 70, 1423-31.

Kwoh, C., Shannon, M. B., Miner, J. H. \& Shaw, A. 2006. Pathogenesis of nonimmune glomerulopathies. Annu Rev Pathol, 1, 349-74.

Le Hir, M., Keller, C., Eschmann, V., Hahnel, B., Hosser, H. \& Kriz, W. 2001. Podocyte bridges between the tuft and Bowman's capsule: an early event in experimental crescentic glomerulonephritis. J Am Soc Nephrol, 12, 2060-71.

Lee, E. Y., Chung, C. H., Khoury, C. C., Yeo, T. K., Pyagay, P. E., Wang, A. \& Chen, S. 2009. The monocyte chemoattractant protein-1/CCR2 loop, inducible by TGF-beta, increases podocyte motility and albumin permeability. Am J Physiol Renal Physiol, 297, F85-94.

Lee, M. J., Feliers, D., Mariappan, M. M., Sataranatarajan, K., Mahimainathan, L., Musi, N., Foretz, M., Viollet, B., Weinberg, J. M., Choudhury, G. G. \& Kasinath, B. S. 2007. A role for AMP-activated protein kinase in diabetes-induced renal hypertrophy. Am J Physiol Renal Physiol, 292, F617-27.

Leeuwis, J. W., Nguyen, T. Q., Dendooven, A., Kok, R. J. \& Goldschmeding, R. 2010. Targeting podocyte-associated diseases. Adv Drug Deliv Rev, 62, 1325-36.

Letavernier, E., Bruneval, P., Mandet, C., Van Huyen, J.-P. D., Pã@Raldi, M.-N. L., Helal, I., Noã«L, L.-H. L. N. \& Legendre, C. 2007. High Sirolimus Levels May Induce Focal Segmental Glomerulosclerosis De Novo. Clinical Journal of the American Society of Nephrology, 2, 326-333.

Liu G, K. B., Kurfis J, Rahmanuddin S, Kanwar YS, Chugh SS 2003. Neph1 and nephrin interaction in the slit diaphragm is an important determinant of glomerular permeability. J Clin Invest, 112, 209-221.

Lloberas, N., Cruzado, J. M., Franquesa, M., Herrero-Fresneda, I., Torras, J., Alperovich, G., Rama, I., Vidal, A. \& Grinyo, J. M. 2006. Mammalian target of rapamycin pathway 
blockade slows progression of diabetic kidney disease in rats. J Am Soc Nephrol, 17, 1395-404.

Loewith, R. 2011. A brief history of TOR. Biochem Soc Trans, 39, 437-42.

Loewith R, J. E., Wullschleger S, Lorberg A, Crespo JL, Bonenfant D, Oppliger W, Jenoe P, Hall MN 2002. Two TOR complexes, only one of which is rapamycin sensitive, have distinct roles in cell growth control. Molecular Cell, 10, 457-468.

Marshall, C. B. \& Shankland, S. J. 2007. Cell cycle regulatory proteins in podocyte health and disease. Nephron Exp Nephrol, 106, e51-9.

Medapalli, R. K., He, J. C, Klotman, P. E. 2011. HIV-associated nephropathy: pathogenesis. Curr Opin Nephrol Hypertens, 20, 306-11.

Meijer, A. J. \& Codogno, P. 2004. Regulation and role of autophagy in mammalian cells. Int J Biochem Cell Biol, 36, 2445-62.

Moeller, M. J., Soofi, A., Hartmann, I., Le Hir, M., Wiggins, R., Kriz, W. \& Holzman, L. B. 2004. Podocytes populate cellular crescents in a murine model of inflammatory glomerulonephritis. J Am Soc Nephrol, 15, 61-7.

Moller, C. C., Flesche, J. \& Reiser, J. 2009. Sensitizing the Slit Diaphragm with TRPC6 ion channels. J Am Soc Nephrol, 20, 950-3.

Mori H, I. K., Masutani K, Wakabayashi Y, Komai K, Nakagawa R, Guan KL, Yoshimura A 2009. The mTOR pathway is highly activated in diabetic nephropathy and rapamycin has a strong therapeutic potential. Biochem Biophys Res Commun, 384, 471-475.

Mundel, P. \& Kriz, W. 1995. Structure and function of podocytes: an update. Anat Embryol (Berl), 192, 385-97.

Mundel, P. \& Shankland, S. J. 2002. Podocyte biology and response to injury. J Am Soc Nephrol, 13, 3005-15.

Munivenkatappa, R., Haririan, A., Papadimitriou, J. C., Drachenberg, C. B., Dinits-Pensy, M. \& Klassen, D. K. 2010. Tubular epithelial cell and podocyte apoptosis with de novo sirolimus based immunosuppression in renal allograft recipients with DGF. Histol Histopathol, 25, 189-96.

Murakami M, I. T., Maeda M, Oshiro N, Hara K, Edenhofer F, Kiyama H, Yonezawa K, Yamanaka S 2004. mTOR is essential for growth and proliferation in early mouse embryos and embryonic stem cells. Mol Cell Biol, 24, 6710-6718.

Nagai, K., Matsubara, T., Mima, A., Sumi, E., Kanamori, H., Iehara, N., Fukatsu, A., Yanagita, M., Nakano, T., Ishimoto, Y., Kita, T., Doi, T. \& Arai, H. 2005. Gas6 induces Akt/mTOR-mediated mesangial hypertrophy in diabetic nephropathy. Kidney Int, 68, 552-61.

Naumovic, R., Jovovic, D., Basta-Jovanovic, G., Miloradovic, Z., Mihailovic-Stanojevic, N., Aleksic, T. \& Jovanovic, D. 2007. Effects of rapamycin on active Heymann nephritis. Am J Nephrol, 27, 379-89.

Orlando Ra, T. T., Zak B, Schmieder S, Benoit VM, Mcquistan T, Furthmayr H, Farquhar MG 2001. The glomerular epithelial cell anti-adhesin podocalyxin associates with the actin cytoskeleton through interactions with ezrin. J Am Soc Nephrol, 12, 1589-1598. 
Patel, P., Pal, S., Ashley, C., Sweny, P. \& Burns, A. 2005. Combination therapy with sirolimus (rapamycin) and tacrolimus (FK-506) in treatment of refractory minimal change nephropathy, a clinical case report. Nephrol Dial Transplant, 20, 985-7.

Pavenstadt, H., Kriz, W. \& Kretzler, M. 2003. Cell biology of the glomerular podocyte. Physiol Rev, 83, 253-307.

Perysinaki GS, M. D., Bertsias G, Giannopoulou I, Kyriakou K, Nakopoulou L, Boumpas DT, Daphnis E 2011. Podocyte main slit diaphragm proteins, nephrin and podocin, are affected at early stages of lupus nephritis and correlate with disease histology. Lupus.

Pollak, M. R. 2008. Focal segmental glomerulosclerosis: recent advances. Curr Opin Nephrol Hypertens, 17, 138-142.

Rangan, G. K. \& Coombes, J. D. 2007. Renoprotective effects of sirolimus in non-immune initiated focal segmental glomerulosclerosis. Nephrol Dial Transplant, 22, 2175-82.

Reiser, J., Oh, J., Shirato, I., Asanuma, K., Hug, A., Mundel, T. M., Honey, K., Ishidoh, K., Kominami, E., Kreidberg, J. A., Tomino, Y. \& Mundel, P. 2004. Podocyte migration during nephrotic syndrome requires a coordinated interplay between cathepsin L and alpha3 integrin. J Biol Chem, 279, 34827-32.

Reiser J, O. J., Shirato I, Asanuma K, Hug A, Mundel TM, Honey K, Ishidoh K, Kominami E, Kreidberg JA, Tomino Y, Mundel P 2004. Podocyte migration during nephrotic syndrome requires a coordinated interplay between cathepsin $\mathrm{L}$ and alpha3 integrin. J Biol Chem, 279, 34827-34832.

Sabatini, D. M. 2006. mTOR and cancer: insights into a complex relationship. Nat Rev Cancer, 6, 729-34.

Sabatini, D. M., Erdjument-Bromage, H., Lui, M., Tempst, P. \& Snyder, S. H. 1994. RAFT1: a mammalian protein that binds to FKBP12 in a rapamycin-dependent fashion and is homologous to yeast TORs. Cell, 78, 35-43.

Sakaguchi, M., Isono, M., Isshiki, K., Sugimoto, T., Koya, D. \& Kashiwagi, A. 2006. Inhibition of mTOR signaling with rapamycin attenuates renal hypertrophy in the early diabetic mice. Biochem Biophys Res Commun, 340, 296-301.

Sarbassov, D. D., Ali, S. M., Sengupta, S., Sheen, J. H., Hsu, P. P., Bagley, A. F., Markhard, A. L. \& Sabatini, D. M. 2006. Prolonged rapamycin treatment inhibits mTORC2 assembly and Akt/PKB. Mol Cell, 22, 159-68.

Sataranatarajan, K., Mariappan, M. M., Lee, M. J., Feliers, D., Choudhury, G. G., Barnes, J. L. \& Kasinath, B. S. 2007. Regulation of elongation phase of mRNA translation in diabetic nephropathy: amelioration by rapamycin. Am J Pathol, 171, 1733-42.

Schwarz, K., Simons, M., Reiser, J., Saleem, M. A., Faul, C., Kriz, W., Shaw, A. S., Holzman, L. B. \& Mundel, P. 2001. Podocin, a raft-associated component of the glomerular slit diaphragm, interacts with CD2AP and nephrin. J Clin Invest, 108, 1621-9.

Senior, P. A., Paty, B. W., Cockfield, S. M., Ryan, E. A. \& Shapiro, A. M. 2005. Proteinuria developing after clinical islet transplantation resolves with sirolimus withdrawal and increased tacrolimus dosing. Am J Transplant, 5, 2318-23.

Sever S, A. M., Nankoe SR, Möller CC, Ko D, Wei C, Henderson J, Del Re EC, Hsing L, Erickson A, Cohen CD, Kretzler M, Kerjaschki D, Rudensky A, Nikolic B, Reiser J 
2007. Proteolytic processing of dynamin by cytoplasmic cathepsin $\mathrm{L}$ is a mechanism for proteinuric kidney disease. J Clin Invest, 117, 2095-2104.

Simon, M., Grone, H. J., Johren, O., Kullmer, J., Plate, K. H., Risau, W. \& Fuchs, E. 1995. Expression of vascular endothelial growth factor and its receptors in human renal ontogenesis and in adult kidney. Am J Physiol, 268, F240-50.

Smoyer, W. E. \& Mundel, P. 1998. Regulation of podocyte structure during the development of nephrotic syndrome. J Mol Med, 76, 172-83.

Somlo, S. \& Mundel, P. 2000. Getting a foothold in nephrotic syndrome. Nat Genet, 24, 333-5.

Succar L, Lai-Kwon J, Nikolic-Paterson D. \& Rangan, G.K. 2007. Inhibition of mTOR reduces crescent formation and improves renal function in experimental crescentic glomerulonephritis (abstract). J Am Soc Nephrol 18, 399A

Sun, D., Toan, X., Zhang, Y., Chen, Y., Lu, R., Wang, X. \& Fang, J. 2008. Mammalian target of rapamycin pathway inhibition enhances the effects of 5-aza-dC on suppressing cell proliferation in human gastric cancer cell lines. Sci China C Life Sci, 51, 640-7.

Thorner, P. S., Ho, M., Eremina, V., Sado, Y. \& Quaggin, S. 2008. Podocytes contribute to the formation of glomerular crescents. J Am Soc Nephrol, 19, 495-502.

Torras J, H.-F. I., Gulias O, Flaquer M, Vidal A, Cruzado JM, Lloberas N, Franquesa M, Grinyó JM 2009. Rapamycin has dual opposing effects on proteinuric experimental nephropathies: is it a matter of podocyte damage? Nephrol Dial Transplant, 24, 36323640 .

Tryggvason, K., Patrakka, J. \& Wartiovaara, J. 2006. Hereditary proteinuria syndromes and mechanisms of proteinuria. $N$ Engl J Med, 354, 1387-401.

Tryggvason K, W. J. 2001. Molecular basis of glomerular permselectivity. Curr Opin Nephrol Hypertens, 10, 543-549.

Tufro, A., Norwood, V. F., Carey, R. M. \& Gomez, R. A. 1999. Vascular endothelial growth factor induces nephrogenesis and vasculogenesis. J Am Soc Nephrol, 10, 2125-34.

Tumlin, J. A., Miller, D., Near, M., Selvaraj, S., Hennigar, R. \& Guasch, A. 2006. A prospective, open-label trial of sirolimus in the treatment of focal segmental glomerulosclerosis. Clin J Am Soc Nephrol, 1, 109-16.

Verma, R., Kovari, I., Soofi, A., Nihalani, D., Patrie, K. \& Holzman, L. B. 2006. Nephrin ectodomain engagement results in Src kinase activation, nephrin phosphorylation, Nck recruitment, and actin polymerization. J Clin Invest, 116, 1346-59.

Vogelbacher, R., Wittmann, S., Braun, A., Daniel, C. \& Hugo, C. 2007. The mTOR inhibitor everolimus induces proteinuria and renal deterioration in the remnant kidney model in the rat. Transplantation, 84, 1492-9.

Vogt, P. K. 2001. PI 3-kinase, mTOR, protein synthesis and cancer. Trends Mol Med, 7, 482-4.

Wang, W., Chan, Y. H., Lee, W. \& Chan, L. 2001. Effect of rapamycin and FK506 on mesangial cell proliferation. Transplant Proc, 33, 1036-7.

Yang, Q. \& Guan, K. L. 2007. Expanding mTOR signaling. Cell Res, 17, 666-81.

Yang, Y., Wang, J., Qin, L., Shou, Z., Zhao, J., Wang, H., Chen, Y. \& Chen, J. 2007. Rapamycin prevents early steps of the development of diabetic nephropathy in rats. Am J Nephrol, 27, 495-502. 
Yaoita, E., Kurihara, H., Yoshida, Y., Inoue, T., Matsuki, A., Sakai, T. \& Yamamoto, T. 2005. Role of Fat1 in cell-cell contact formation of podocytes in puromycin aminonucleoside nephrosis and neonatal kidney. Kidney Int, 68, 542-51.

Zhu, J., Sun, N., Aoudjit, L., Li, H., Kawachi, H., Lemay, S. \& Takano, T. 2008. Nephrin mediates actin reorganization via phosphoinositide 3-kinase in podocytes. Kidney Int, 73, 556-66. 


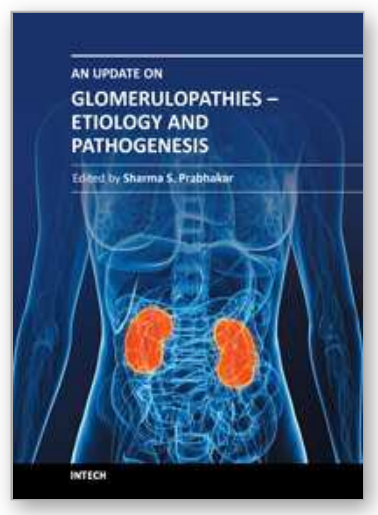

\author{
An Update on Glomerulopathies - Etiology and Pathogenesis \\ Edited by Prof. Sharma Prabhakar
}

ISBN 978-953-307-388-0

Hard cover, 276 pages

Publisher InTech

Published online 06, September, 2011

Published in print edition September, 2011

The book has fourteen chapters which are grouped under different sections: Immune System and Glomerulonephritis, Animal Models of Glomerulonephritis, Cytokines and Signalling Pathways, Role of Cells and Organelles in Glomerulonephritis and Miscellaneous. While the purpose of this volume is to serve as an update on recent advances in the etio-pathogenesis of glomerulopathies, the book offers the current and broad based knowledge in the field to readers of all levels in the nephrology community.

\title{
How to reference
}

In order to correctly reference this scholarly work, feel free to copy and paste the following:

Lena Succar, David CH Harris and Gopala K Rangan (2011). Role of the Mammalian Target of Rapamycin (mTOR) Signalling Pathway in Podocytes in Glomerular Disease, An Update on Glomerulopathies - Etiology and Pathogenesis, Prof. Sharma Prabhakar (Ed.), ISBN: 978-953-307-388-0, InTech, Available from:

http://www.intechopen.com/books/an-update-on-glomerulopathies-etiology-and-pathogenesis/role-of-themammalian-target-of-rapamycin-mtor-signalling-pathway-in-podocytes-in-glomerular-disease

\section{INTECH}

open science | open minds

\section{InTech Europe}

University Campus STeP Ri

Slavka Krautzeka 83/A

51000 Rijeka, Croatia

Phone: +385 (51) 770447

Fax: +385 (51) 686166

www.intechopen.com

\section{InTech China}

Unit 405, Office Block, Hotel Equatorial Shanghai

No.65, Yan An Road (West), Shanghai, 200040, China

中国上海市延安西路65号上海国际贵都大饭店办公楼 405 单元

Phone: +86-21-62489820

Fax: +86-21-62489821 
(C) 2011 The Author(s). Licensee IntechOpen. This chapter is distributed under the terms of the Creative Commons Attribution-NonCommercialShareAlike-3.0 License, which permits use, distribution and reproduction for non-commercial purposes, provided the original is properly cited and derivative works building on this content are distributed under the same license. 